\title{
Kinetics of sorption in hygroscopic hydrogels
}

3

$6{ }^{1}$ Department of Mechanical Engineering, Massachusetts Institute of Technology, Cambridge,

7 Massachusetts 02139, United States

$8{ }^{2}$ Department of Materials Science and Engineering, Massachusetts Institute of Technology,

9 Cambridge, Massachusetts 02139, United States

$10 \quad{ }^{\#}$ Equal contribution to this work

11

12

13

14

15

16

17

Carlos D. Díaz-Marín ${ }^{1, \#}$, Lenan Zhang ${ }^{1, \#, *}$, Zhengmao Lu², Mohammed Alshrah ${ }^{1}$, Jeffrey C.
Grossman ${ }^{2}$, Evelyn N. Wang

\author{
-
}




\section{Derivation of vapor transport equation}

19 We consider mass conservation for vapor and liquid phases inside the hydrogel to derive the

20 governing equations for hydrogel absorption. Specifically, we consider one-dimensional mass

21 transport (considering a thin hydrogel layer) and consider the hydrogel as a homogeneous porous

22 medium composed of three phases: solid (i.e. polymer matrix), liquid and gaseous with volume

23 fractions $\phi_{\mathrm{s}}, \phi_{1}$, and $\phi_{\mathrm{g}}$, respectively.

24 For the conservation of vapor, we consider a differential control volume $d V$ (Figure S1) with

25 length in the $\mathrm{x}$ direction $d x$ and cross-sectional area $d A$, such that $d V=d A d x$. We describe vapor

26 content in terms of the number of moles of vapor per volume of air $(C)$. Therefore, the number of

27 moles of vapor inside the control volume is $C \phi_{\mathrm{g}} d V$. Vapor is diffused into the control volume

28 with a molar flux $J_{\mathrm{C} \text {, }}$ and is diffused out of the $d V$ with a molar flux $J_{\mathrm{C}, \mathrm{x}+\mathrm{dx}}$. Inside the control

29 volume, vapor condenses into liquid at a molar rate $\dot{R} d V$.

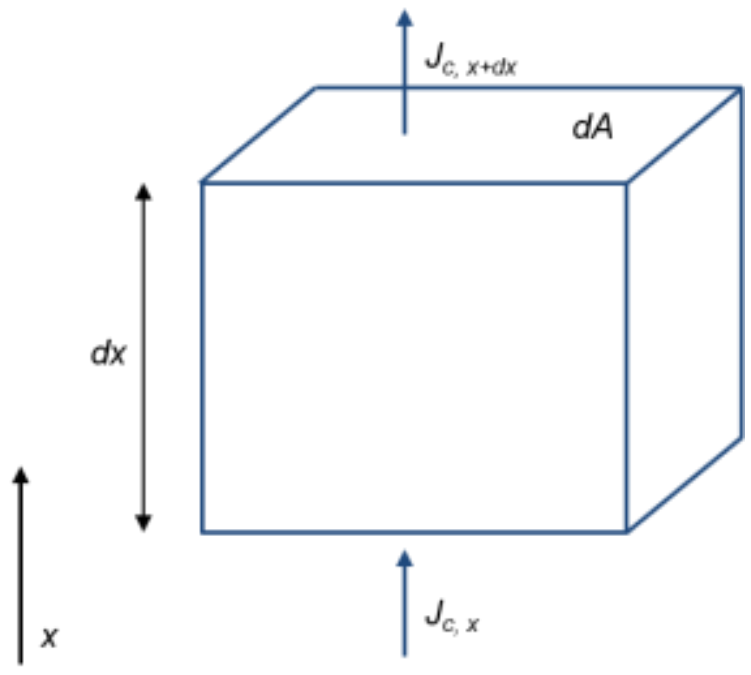

31 Figure S1. Control volume used for the derivation of the governing equations. Vapor diffuses

32 into and out of the differential control volume with fluxes $J_{\mathrm{C}, \mathrm{x}}$ and $J_{\mathrm{C}, \mathrm{x}+\mathrm{dx}}$, respectively 
33 Conservation of vapor inside the control volume will therefore imply that:

$$
\frac{\partial\left(\phi_{\mathrm{g}} C d V\right)}{\partial t}=J_{\mathrm{C}, \mathrm{x}} d A-J_{\mathrm{C}, \mathrm{x}+\mathrm{dx}} d A-\dot{R} d V
$$

34 Dividing Equation (1) by $d V$ and writing $J_{C}=-D_{\text {eff }} \frac{\partial C}{\partial x}$, where $D_{\text {eff }}$ is the effective diffusion 35 coefficient of vapor inside the porous hydrogel, we obtain:

$$
\frac{\partial\left(\phi_{\mathrm{g}} C\right)}{\partial t}-\frac{\partial}{\partial x}\left(D_{\mathrm{eff}} \frac{\partial C}{\partial x}\right)=-\dot{R}
$$

$36 \dot{R}$, the rate at which moles of vapor condense per unit volume, is given by the considering the

37 condensation inside each micropore of the hydrogel. This condensation is driven by a difference

38 in vapor concentration between the liquid-vapor interface and the pore. The liquid-vapor

39 interface will have a vapor concentration of $\frac{a_{\mathrm{w}, \mathrm{s}} P_{\mathrm{sat}}}{R T}$, where $a_{\mathrm{w}, \mathrm{s}}$ is the activity of the water-salt

40 mixture, $T$ is the temperature, $R$ is the gas constant, and $P_{\text {sat }}$ is the vapor saturation pressure at a

41 temperature $T$. Therefore, inside each pore, condensation occurs at a rate $-S D_{v}\left(\frac{a_{\mathrm{w}, \mathrm{s}} P_{\mathrm{sat}}}{R T}-C\right){ }^{1}$

42 where $S$ is the shape factor for the diffusion inside the pore (see Section S4 for details) and $D_{v}$ is

43 the diffusion coefficient of vapor in air. If there are $N$ micropores per volume, then Equation S2

44 can be written as:

$$
\frac{\partial\left(\phi_{\mathrm{g}} C\right)}{\partial t}-\frac{\partial}{\partial x}\left(D_{\mathrm{eff}} \frac{\partial C}{\partial x}\right)=N S D_{v}\left(\frac{a_{\mathrm{w}, \mathrm{s}} P_{\mathrm{sat}}}{R T}-C\right)
$$

45 which corresponds to Equation 1 in the main text. It should be noted that in deriving Equation $46 \mathrm{~S} 3$, it was considered that the saturation pressure is unchanged by the curvature of the pores 47 where condensation happens. This is the case since the hydrogel pores have micron-sized radii 
48 which are significantly larger than the nanometer-sized radii necessary for the Kelvin effect to be

49 significant. $^{2}$

\section{Derivation of liquid transport equation}

51 The governing equation for liquid transport is also derived by considering mass conservation for

52 a differential control volume $d V$. We describe liquid content with $n$, the molar concentration per

53 initial volume of the gel (i.e. initial volume of polymer and liquid). Therefore, the number of

54 moles inside the control volume is $n\left(1-\phi_{\mathrm{g} 0}\right) d V$, where $\phi_{\mathrm{g} 0}$ is the initial volume fraction of gas in

55 the hydrogel. Liquid is diffused into and out of the control volume with molar fluxes $J_{\mathrm{n}, \mathrm{x}}$ and

$56 J_{\mathrm{n}, \mathrm{x}+\mathrm{dx}}$. Liquid is generated inside the control volume at a rate $\dot{R} d V$ due to condensation of vapor,

57 with $\dot{R}=-N S D_{v}\left(\frac{a_{\mathrm{w}, \mathrm{s}} P_{\mathrm{sat}}}{R T}-C\right)$, as derived in Section 1.

58 With this, mass conservation for the control volume implies that

$$
\frac{\partial\left(\left(1-\phi_{\mathrm{g} 0}\right) n\right)}{\partial t}=-\frac{\partial J_{n}}{\partial x}-N S D_{v}\left(\frac{a_{\mathrm{w}, \mathrm{s}} P_{\mathrm{sat}}}{R T}-C\right) .
$$

59 The diffusive flux $J_{\mathrm{n}}$ arises from the differences in water content inside the hydrogel and it can be

60 expressed in terms of the liquid concentration $n$ through Biot's theory of poroelasticity. We

61 specifically follow the derivation from Yoon et al. ${ }^{3}$ First, we consider that diffusion in the porous

62 polymer matrix of the hydrogel follows Darcy's law

$$
J_{n}=-\frac{k \rho_{\mathrm{l}}^{2}}{\eta M^{2}} \frac{\partial \mu}{\partial x}
$$

63 where $k$ is the permeability of the nanoporous polymer. $\eta, M, \rho_{\mathrm{l}}$, and $\mu$ are the viscosity, molar

64 mass, density, and chemical potential of the liquid water inside the hydrogel, respectively. 
65 As the liquid diffuses in the polymer, it generates a volumetric expansion of the hydrogel. If the 66 polymer and water molecules are considered incompressible, the local volumetric deformation of 67 the hydrogel $\sum_{k} \varepsilon_{k k}$ is given by

$$
\sum_{k} \varepsilon_{k k}=\left(n-n_{0}\right) \frac{M}{\rho_{1}}
$$

68 where $n_{0}$ is the initial molar concentration of water inside the hydrogel.

69 The coupled volumetric expansion of the hydrogel and diffusion of water induce a change $d F$ in

70 the Helmholtz free energy per volume of the system

$$
d F=\sum_{i, j} \sigma_{i j} d \varepsilon_{i j}+\mu d n,
$$

71 where $\sigma_{i j}$ and $\varepsilon_{i j}$ are the stress and strain fields inside the hydrogel, respectively. Following a 72 linear treatment of the hydrogel, the Helmholtz free energy of the system is taken to be quadratic 73 in the strain and dependent on the shear modulus $G$ and the Poisson ratio $v$

$$
W=G\left(\sum_{i, j} \varepsilon_{i j}^{2}+\frac{v}{1-2 v} \sum_{i} \varepsilon_{i i}^{2}\right)
$$

74 Combining equations S6, S7, S8

$$
\sigma_{i j}=2 G\left(\varepsilon_{i j}+\frac{v}{1-2 v} \delta_{i j} \sum_{k} \varepsilon_{k k}\right)-\mu \frac{\rho_{1}}{M} \delta_{i j}
$$


75 where $\delta_{i j}$ is the Kronecker delta $\left(\delta_{i j}=1\right.$ for $i=j, \delta_{i j}=0$ for $\left.i \neq j\right)$. Additionally, considering

76 the hydrogel in mechanical equilibrium $\left(\frac{\partial \sigma_{i j}}{\partial x_{j}}=0\right)$, using equations S6 and S9, and assuming

77 that the hydrogel differential material elements stretch isotropically in all directions (i.e. $\varepsilon_{x x}=$

$78 \varepsilon_{y y}=\varepsilon_{z z}$ ), the gradient of the chemical potential can be expressed as

$$
\frac{\partial \mu}{\partial x}=\frac{M^{2}}{3 \rho_{1}^{2}} \frac{2 G(1+v)}{(1-2 v)} \frac{\partial n}{\partial x}
$$

79 and, combining with equations $\mathrm{S} 4$ and $\mathrm{S} 5$, this leads to the desired diffusion equation

$$
\left(1-\phi_{\mathrm{g} 0}\right) \frac{\partial n}{\partial t}-\frac{\partial}{\partial x}\left(D_{l} \frac{\partial n}{\partial x}\right)=-N S D_{v}\left(\frac{a_{\mathrm{w}, \mathrm{s}} P_{\mathrm{sat}}}{R T}-C\right)
$$

80 with the effective liquid diffusion coefficient $D_{l}$ given by

$$
D_{1}=\frac{2(1+v) G}{3(1-2 v) \eta} k
$$

81 Our derived diffusion coefficient differs slightly from that of Yoon $e t a l^{3}$ since we assumed that

82 the hydrogel deforms identically in all directions. This deformation in the $\mathrm{x}$ direction results in

83 an overall increase in the hydrogel thickness, while if the hydrogel is constrained in the other two

84 directions, the gel deformation occurs at the expense of gas volume within the hydrogel.

\section{3. Equations for the volume fractions}

86 As the hydrogel captures water from the vapor, the volume fractions of solid, liquid, and gas

87 within the hydrogel change. As such, we can express these volume fractions as functions of the

88 water concentration $n$. For this, we write the volume for the entire hydrogel system (i.e. the

89 system composed of gas, polymer, and water volume fractions) 


$$
V_{\text {tot }}=V_{\text {tot }, 0}\left(1+\varepsilon_{x x}\right) \text {, }
$$

90 where it was assumed that the hydrogel is constrained in the $\mathrm{y}$ and $\mathrm{z}$ direction and it expands

91 freely in the $\mathrm{x}$ direction. Even though the entire hydrogel is constrained in the $\mathrm{y}$ and $\mathrm{z}$ directions,

92 the individual hydrogel differential material elements deform isotropically in the three directions,

93 where the deformation in the $y$ and $\mathrm{z}$ directions comes at the expense of pore volume. Therefore,

94 from Equation S6

$$
\varepsilon_{x x}=\varepsilon_{y y}=\varepsilon_{z z}=\frac{\left(n-n_{0}\right) M}{3 \rho_{1}}
$$

95 The volumes of the solid, liquid, and gas phases are, and respectively,

$$
\begin{gathered}
V_{\mathrm{s}}=V_{\mathrm{tot}, 0} \phi_{\mathrm{s}, 0}, \\
V_{\mathrm{l}}=V_{\mathrm{tot}, 0}\left(1-\phi_{\mathrm{g}, 0}\right) n \frac{M}{\rho_{\mathrm{l}}} \\
V_{\mathrm{g}}=V_{\mathrm{tot}}-V_{\mathrm{s}}-V_{\mathrm{l}},
\end{gathered}
$$

96 where it was once again assumed that the polymer and water are incompressible.

97 As a consequence, the volume fractions of solid, liquid, and gas can be written as

$$
\begin{gathered}
\phi_{\mathrm{s}}=\frac{V_{\mathrm{s}}}{V_{\mathrm{tot}}}=\frac{\phi_{\mathrm{s}, 0}}{\left(1+\varepsilon_{x x}\right)}, \\
\phi_{\mathrm{l}}=\frac{V_{\mathrm{l}}}{V_{\mathrm{tot}}}=\frac{n M\left(1-\phi_{g, 0}\right)}{\rho_{\mathrm{l}}\left(1+\varepsilon_{x x}\right)}, \\
\phi_{\mathrm{g}}=\frac{V_{\mathrm{g}}}{V_{\mathrm{tot}}}=1-\phi_{\mathrm{s}}-\phi_{\mathrm{l}} .
\end{gathered}
$$


98 In our model, we have treated the solid to be composed of salt and polymer. This introduces a

99 small error since once the salt deliquesces it will take up a different volume from its solid

100 volume. This error is small because the salt occupies only a small portion of the entire hydrogel.

101 For instance, once the hydrogel reaches equilibrium the volume of salt can be as low as $\sim 2 \%$ of

102 the entire volume.

\section{4. Activity function}

104 We developed a polynomial fit (Figure S2) to approximate the experimental data for the activity 105 of water in a calcium chloride aqueous solution. ${ }^{4-7}$ The polynomial fit of Figure S2 captures the 106 entire sorption process driven by salt. First, salt bonds with water to form hydrates at high salt 107 mass fractions $(>0.5)$. Once the salt hydrates have reached their maximum hydration state they 108 can continue capturing water by dissolution.

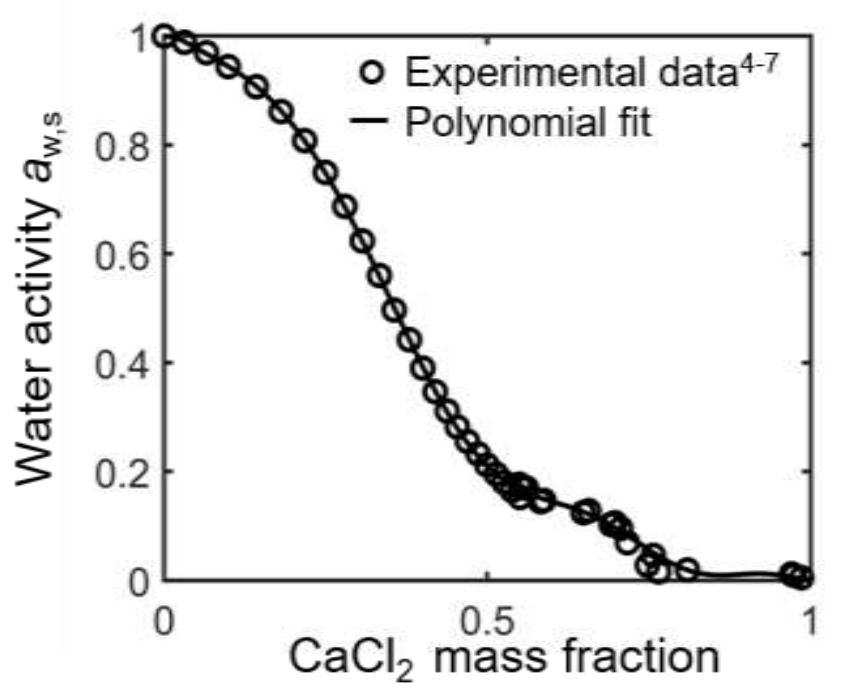

110 Figure S2. Polynomial fit developed to approximate literature data for the water activity in 111 calcium chloride and water system. 
112 In our simulation, the $\mathrm{CaCl}_{2}$ mass fraction was calculated as

$$
\mathrm{CaCl}_{2} \text { mass fraction }(x, t)=\frac{n_{\text {salt }} M_{\text {salt }}}{n(x, t) M+n_{\text {salt }} M_{\text {salt }}},
$$

113 where $n_{\text {salt }}$ is the molar concentration of salt per unit of dry hydrogel volume and $M_{\text {salt }}$ is the

114 molar mass of salt. As the water concentration changes with position and time, the salt molar

115 concentration is also a spatially and temporally varying variable. The evolution of salt mass

116 fraction leads to a reduction in the sorption driving force $\left(\frac{a_{\mathrm{w}, \mathrm{s}} P_{\mathrm{sat}}}{R T}-C\right)$. The system reaches the

117 equilibrium state when this sorption driving force term approaches zero.

\section{5. Shape factor for a pore}

119 The vapor transport in a single micropore is described by the $1 \mathrm{D}$ diffusion equation in spherical

120 coordinates,

$$
\frac{1}{r^{2}} \frac{d}{d r}\left(r^{2} \frac{d c}{d r}\right)=0
$$

121 where $c$ is the microscopic vapor concentration in the micropore. Eq. (S20) has a general

122 solution expressed as,

$$
c=\frac{a}{r}+b
$$

123 where $a$ and $b$ are two constants determined by the boundary conditions of the system. Since

124 vapor on the wall of the micropore (i.e., the liquid-gas interface) is in the saturation condition, its 125 concentration is determined by the saturation vapor concentration $C_{\text {sat, }}$,

$$
C_{\mathrm{sat}}=c\left(r_{p}\right)=\frac{a}{r_{\mathrm{p}}}+b
$$


126 where $r_{\mathrm{p}}$ is the micropore radius. The microscopic concentration is related to the macroscopic

127 concentration by performing volumetric average throughout the entire micropore,

$$
C=\frac{1}{\frac{4}{3} \pi r_{\mathrm{p}}^{3}} \int_{0}^{r_{p}} c \times 4 \pi r^{2} d r=\frac{3}{2} \frac{a}{r_{\mathrm{p}}}+b .
$$

128 The vapor flow across the wall of micropore $J$ is thus given by,

$$
J=\left(-4 \pi r^{2} D_{\mathrm{v}} \frac{d c}{d r}\right)_{r=r_{\mathrm{p}}}=8 \pi r_{\mathrm{p}} D_{\mathrm{v}}\left(C-C_{\mathrm{sat}}\right)=S D_{\mathrm{v}}\left(C-C_{\mathrm{sat}}\right) .
$$

129 Therefore, the shape factor of a micropore $S$ is given by,

$$
S=8 \pi r_{\mathrm{p}}
$$

130 This shape factor $S$ will be a function of time and position since the micropore radius changes as 131 water condenses into the hydrogel. Specifically, the pore radius and the gas volume fraction are 132 related by

$$
N\left(\frac{4 \pi r_{\mathrm{p}}^{3}}{3}\right)=\phi_{g}
$$

\section{6. Model assumptions}

134 We have made several assumptions in our model development. Here we list these assumptions.

135 a) Transport in the hydrogel is in $1 \mathrm{D}$.

136 b) The hydrogel contributes negligibly to the equilibrium water uptake. The driving force of 137 sorption is determined by the sorbents only. ${ }^{8,9}$ 
138 c) The hydrogel was considered to be a random porous media and therefore the tortuosity of 139 the pores is given by $\phi^{-1 / 2} \cdot 10,11$

140 d) Pores in the hydrogel remain spherical at all times.

141 e) Each differential material element of the hydrogel deforms isotropically. The entire 142 hydrogel is constrained to expand only in one direction (the $\mathrm{x}$ direction in Fig. 1). This 143 implies that the deformation of differential material elements of the hydrogel along the other 144 two directions comes at the expense of pore volume.

145 f) The hydrogel exhibits a linear poroelastic behavior. ${ }^{3,12}$

$146 \mathrm{~g}$ ) The system is considered to be isothermal at the same temperature of the ambient (see 147 Section S14 for details).

\section{Properties of hydrogel used in simulation}

149 Table S1. Properties of hydrogel used in simulation

\begin{tabular}{|c|c|}
\hline Property & Value \\
\hline Initial thickness $H$ & $5 \mathrm{~mm}^{9}$ \\
\hline Dried state solid volume fraction $\phi_{\mathrm{s} 0}$ & 0.1 \\
\hline Initial micropore radius $r_{\mathrm{p}}$ & $1 \mu \mathrm{m}^{9}$ \\
\hline Pore density $N$ & $\phi_{\mathrm{s} 0} /\left(4 \pi \mathrm{r}_{\mathrm{p}}{ }^{3 / 3}\right)$ \\
\hline Shear modulus $G$ & $1000 \mathrm{~Pa}^{13}$ \\
\hline Poisson ratio & $0.45^{14}$ \\
\hline Density polyacrylamide & $1300 \mathrm{~kg}^{13} \mathrm{~m}^{315}$ \\
\hline Salt content & $4 \mathrm{~g} / \mathrm{g} \mathrm{dry} \mathrm{hydrogel}^{9}$ \\
\hline
\end{tabular}




\section{Boundary conditions}

151 Equations 1 and 2 from the main text were solved subject to the following boundary conditions:

$$
\begin{gathered}
\left.\frac{\partial C}{\partial x}\right|_{x=0}=0, \\
\left.C\right|_{x=H}=R H \cdot \frac{P_{\mathrm{sat}}}{R T}, \\
\left.\frac{\partial n}{\partial x}\right|_{x=0}=0, \\
\left.\frac{\partial n}{\partial x}\right|_{x=H}=0
\end{gathered}
$$

152 where $R H$ is the ambient relative humidity. In the previous equations, it was assumed that the

153 bottom layer was insulated from the ambient so there is no diffusion at $x=0$ (Eq. S29). In the

154 top layer, the hydrogel was considered in equilibrium with the ambient and, therefore, the vapor

155 concentration is $R H \cdot \frac{P_{\text {sat }}}{R T}(\mathrm{Eq}$. S30). It was assumed that there was no liquid transport into or out 156 of the hydrogel directly in the top and bottom (Eq. S31 and S32).

\section{9. Calculation of permeability and pore size}

158 The permeability of the nanoporous network $k$ was taken as a function of the liquid volume 159 fraction $\phi_{1}$

$$
k=\frac{k_{0} \phi_{1}}{\left(1-\phi_{1}\right)^{1.5}}
$$


160 and, as such will be a function of time and position. Figure S3 shows the local permeability 161 plotted as a function of distance and for different times after the start of the sorption for an 162 ambient humidity of $80 \%$. The nanoporous permeability will be higher when there is a higher 163 water concentration as captured by equations S18 and S31, reaching a maximum value of about $1641.5 \times 10^{-17} \mathrm{~m}^{2}$.

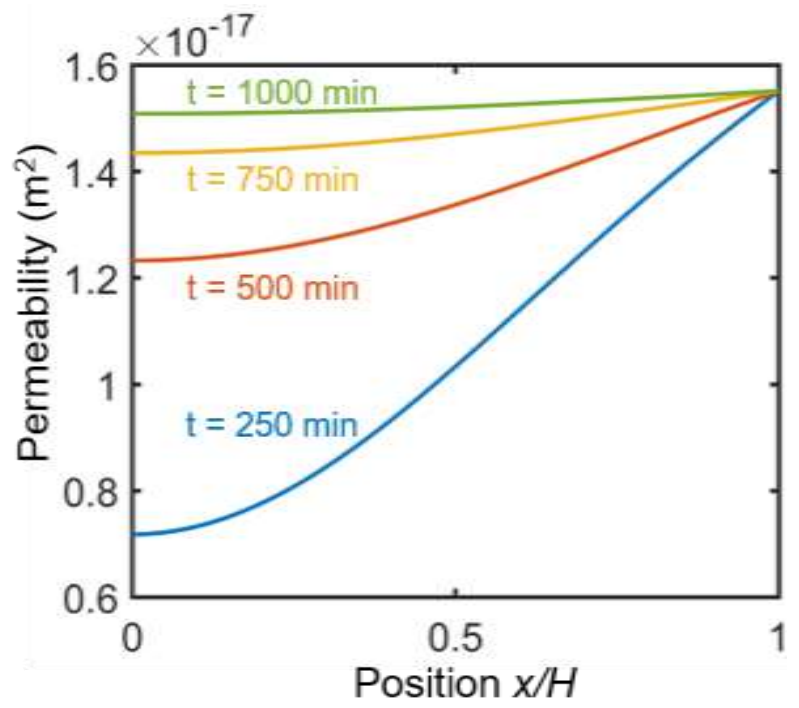

166 Figure S3. Nanoporous network permeability as a function of time and position. Permeability 167 will be higher in places with high water concentration.

168 The effective pore diameter $d$ of the polymer matrix can be calculated form the permeability. 169 Assuming that the pores are straight cylinders with diameter $d$, then ${ }^{16}$

$$
k=\frac{d^{2} \phi_{1}}{32},
$$

170 which allows to calculate the effective pore diameter from the permeability. Owing to the spatial 171 and time dependence of the permeability and the liquid volume fraction, the effective pore 
172 diameter, as defined by equation S31, will also be time and space dependent, as shown in Figure

173 S4 and will have a value of $22-29 \mathrm{~nm}$.

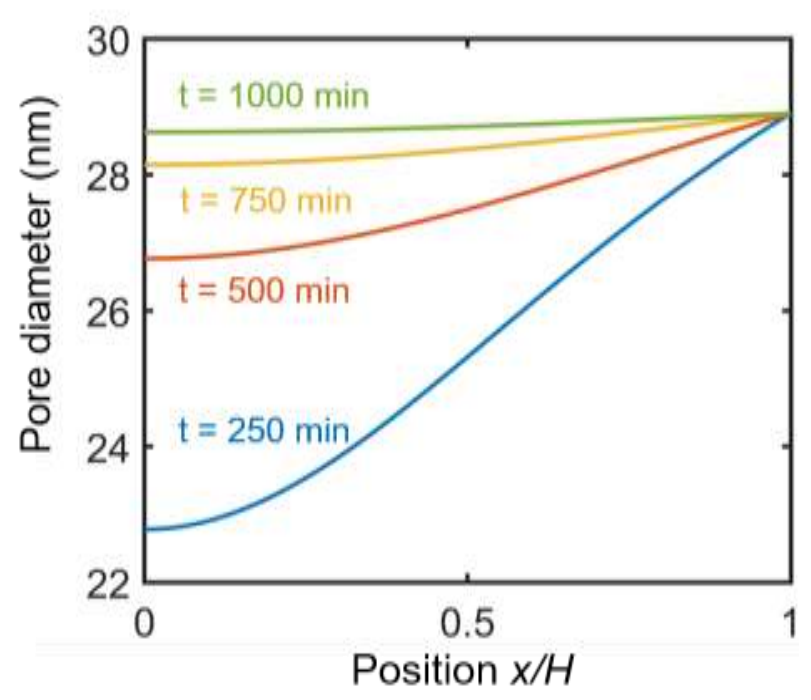

175 Figure S4. Effective pore diameter of the polymer matrix as a function of time and position.

176 10. Exponential fitting for the uptake curves

177 The curves obtained from our model were approximated to exponential functions that tend to the 178 equilibrium uptake Uptake $_{\infty}$, that is

$$
\text { Uptake }=\text { Uptake }_{\infty}\left(1-e^{-\frac{t}{\tau}}\right) .
$$

179 Based on this, we can define a dimensionless uptake $\widetilde{U}=\left(1-\text { Uptake/Uptake }_{\infty}\right)^{-1}$ whose

180 logarithm is a linearly increasing function of time, with a slope equal to $\tau$. For each of the uptake 181 curves, the timescale $\tau$ was found by fitting the uptake curves from the model to the simplified 182 expression S35. The dimensionless uptake for the model results and the approximation are 
183 shown in Figure S5. The approximate exponential curves are seen to capture accurately the 184 results from the model, providing a method to quantifying the kinetics through $\tau$.
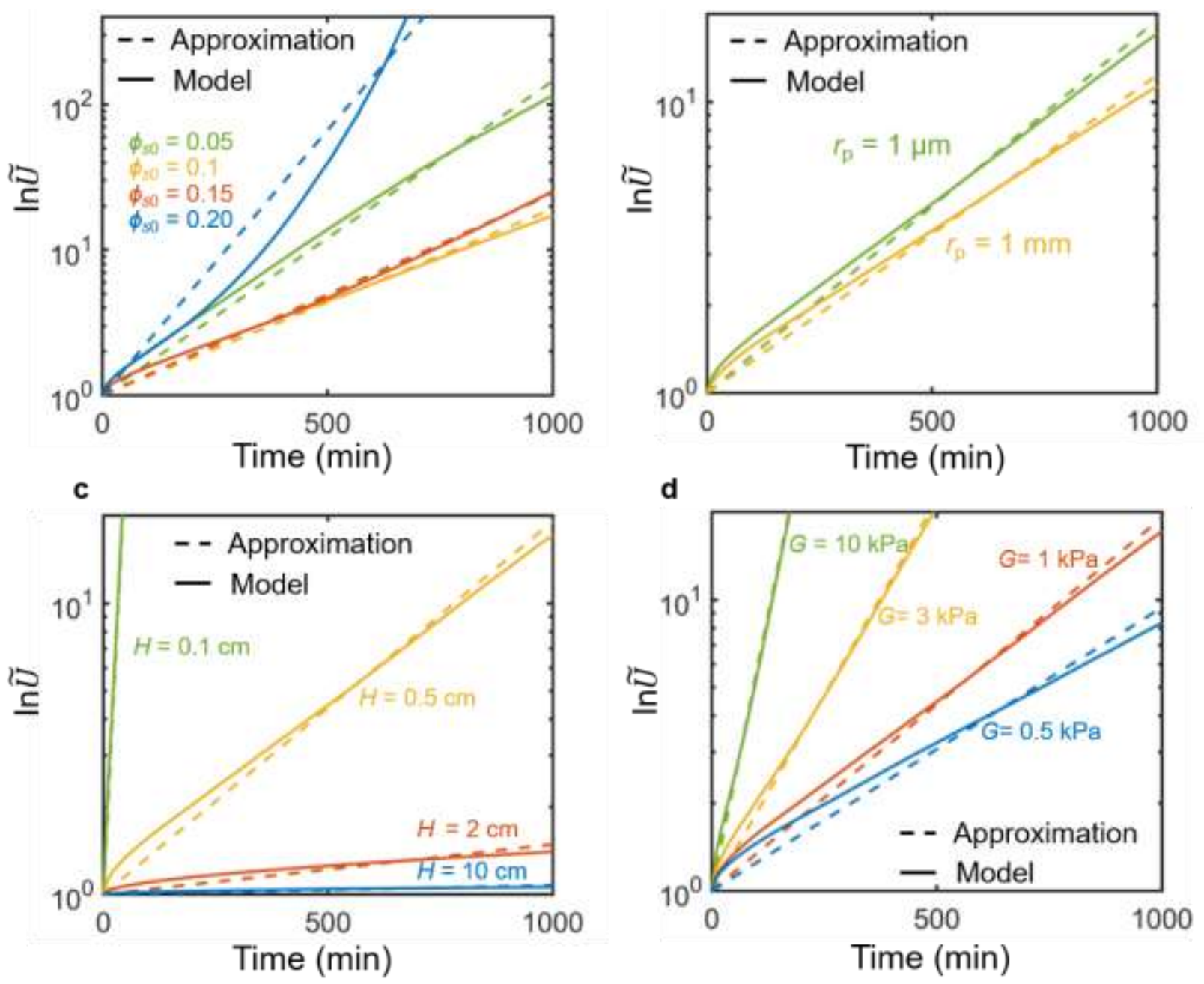

185

186 Figure S5. Dimensionless uptake for the model results and the approximate exponential 187 functions as a function of time for different (a) initial solid volume fractions, (b) initial 188 micropore radius, (c) initial hydrogel thickness, (d) shear modulus. 
191 11. Thickness dependence of the effective timescale

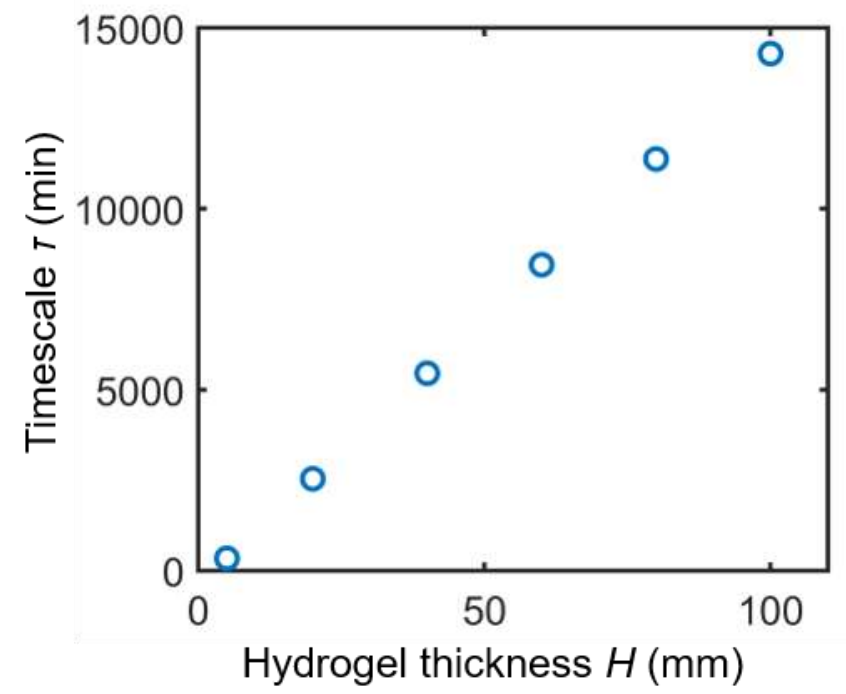

193 Figure S6. Absorption timescale $\tau$ as a function of the hydrogel layer thickness $H$. 


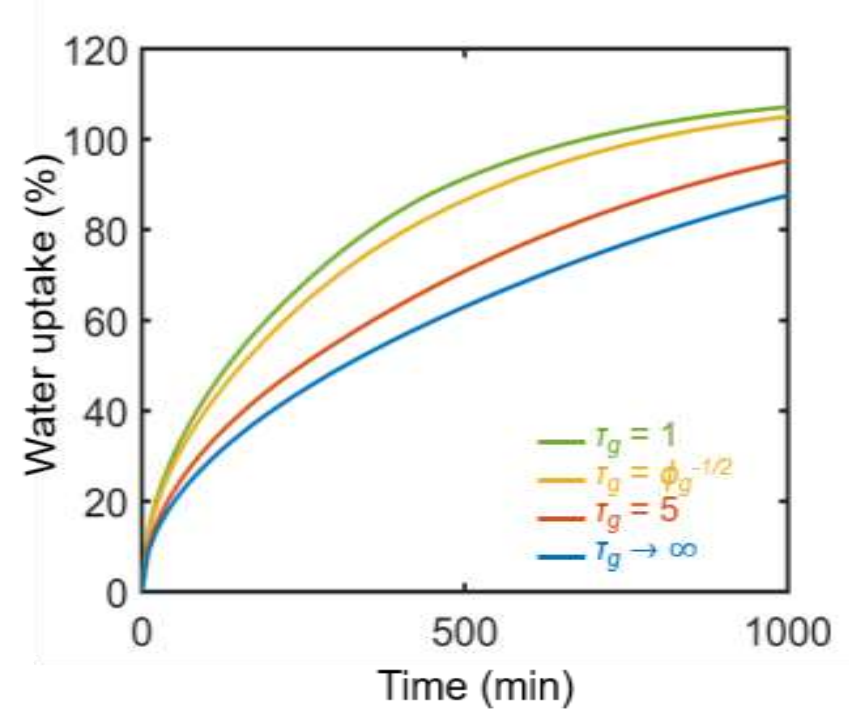

205 Figure S7. Uptake curves for hydrogels with different tortuosities for a relative humidity of $60 \%$,

206 salt loading of $3 \mathrm{~g} / \mathrm{g}$ of polymer, and thickness of $5 \mathrm{~mm}$. The limit of tortuosity approaching

207 infinity corresponds to the case of water sorption without vapor diffusion. 


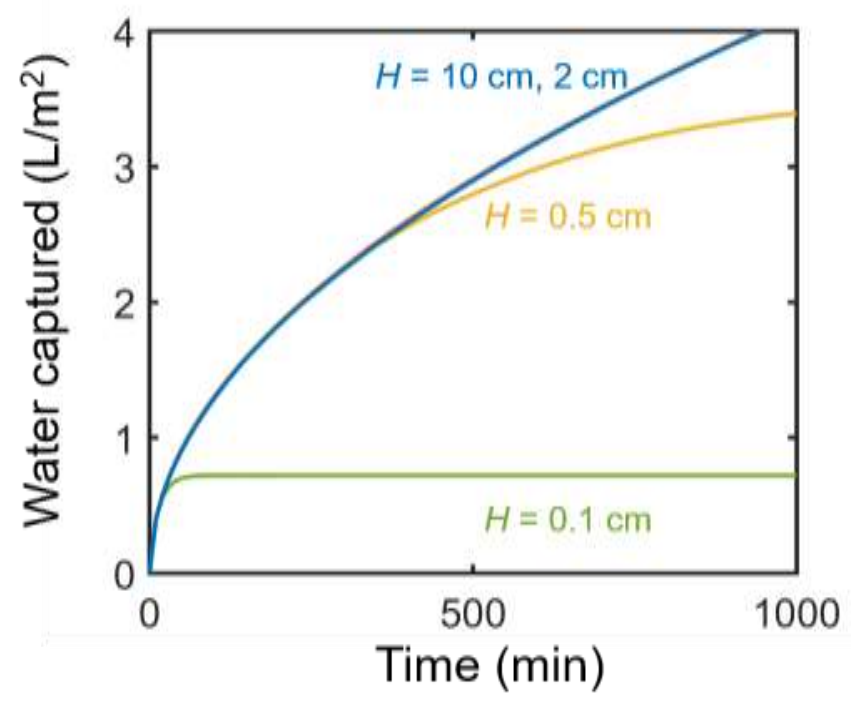

217 Figure S8. Total water captured as a function of time for different thicknesses $H$. The curves for $218 H=2 \mathrm{~cm}$ and $H=10 \mathrm{~cm}$ are identical.

\section{14. Heat generation during sorption}

220 Typically, sorption processes are accompanied by a heat release as water binds to the sorbents.

221 For hygroscopic hydrogels embedded with salts, this heat release is due to water condensation

222 and salt dilution. ${ }^{17,18}$ Here, we perform a simplified simulation that aims to capture the resulting

223 temperatures from the heat release during sorption. We model heat generation and conduction in

224 the hydrogel, where we consider the hydrogel as an effective porous media described by:

$$
k_{\mathrm{eff}} \frac{\partial^{2} T}{\partial x^{2}}+\dot{q}_{\mathrm{gen}}=\left(\rho c_{p}\right)_{\mathrm{eff}} \frac{\partial T}{\partial t}
$$


225 Where $T$ is the temperature and $\dot{q}_{\text {gen }}$ is the volumetric heat generation due to sorption. $k_{\text {eff }}$ is the 226 effective thermal conductivity of the porous media and $\left(\rho c_{p}\right)_{\text {eff }}$ is the effective thermal mass of 227 the media, which are given by:

$$
\begin{gathered}
k_{\mathrm{eff}}=\phi_{l} k_{l}, \\
\left(\rho c_{p}\right)_{\mathrm{eff}}=\rho_{l} c_{p, l} \phi_{l},
\end{gathered}
$$

228 respectively, where $k_{l}, \rho_{l}, c_{p, l}$ are the thermal conductivity, density, and heat capacity of water, 229 respectively. Eqs. S37 and S38 assume that the liquid water dominates the heat transport and 230 thermal mass, due to the low thermal conductivity and thermal mass of air, as well as the small 231 volume fractions of solid. In theory, the volume fraction of liquid water evolves with time and 232 position as water condenses in the hydrogel, as captured by Eq. S19. Equation S19 couples 233 Equation S36 with Eqs. 1 and 2 of the main text. To simplify this highly coupled problem and 234 provide reasonable predictions, we instead considered a constant average liquid volume fraction 235 of $\phi_{l}=0.5$ for our heat transfer analysis. Heat generation is also a function of the rate of water 236 sorption. We performed a similar simplification with a constant heat generation given by

$$
\dot{q}_{\text {gen }}=\frac{\Delta m_{\mathrm{total}} h_{\mathrm{lv}}}{V t_{\mathrm{total}}}
$$

237 where $\Delta m_{\text {total }}$ is the total mass of water that enters the hydrogel system, $V$ is the initial volume 238 of the hydrogel, including the volume of its pores, $t_{\text {total }}$ is the total time of sorption (which is $2391000 \mathrm{~min}$ in our simulation), and $h_{\mathrm{lv}}$ is the latent heat of condensation of water. As a 240 consequence, the heat generation rate given by Eq. S39 corresponds to an average value.

241 Equation S36 was solved subject to the following boundary and initial conditions:

$$
k_{\mathrm{eff}} \frac{\partial T}{\partial x}(x=0, t)=h\left(T-T_{\infty}\right)
$$




$$
\begin{gathered}
-k_{\mathrm{eff}} \frac{\partial T}{\partial x}(x=H, t)=h\left(T-T_{\infty}\right), \\
T(x, t=0)=T_{\infty},
\end{gathered}
$$

242 where $T_{\infty}=22{ }^{\circ} \mathrm{C}$ is the ambient temperature and $h$ is the heat transfer coefficient due to 243 convection and radiation, which is given by ${ }^{19}$

$$
h=h_{\text {conv }}+\varepsilon \sigma\left(T^{2}+T_{\infty}^{2}\right)\left(T+T_{\infty}\right),
$$

244

245 where $h_{\text {conv }}$ is the convection heat transfer coefficient, $\varepsilon$ is the hydrogel emissivity, $\sigma$ is the 246 Stefan Boltzmann constant. For our simulation, we used $h_{\text {conv }}=50 \mathrm{~W} / \mathrm{m}^{2} \mathrm{~K}$, which is a 247 conservative estimate for forced convection in gases ${ }^{19}$ and $\varepsilon=1^{9}$. Our results for Equations S3624843 are shown in Figure S8, where we considered the case of a relative humidity of $80 \%$ and, 249 therefore, the highest mass gain and heat generation.

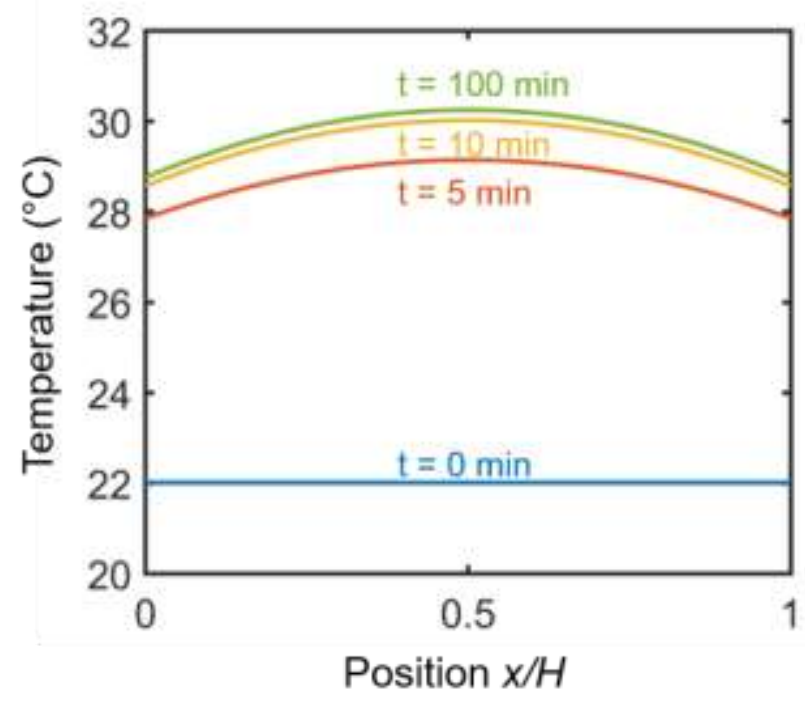

251 Figure S9. Simulation results of the temperature profile in the hydrogel during sorption.

252 As seen in Figure S8, the hydrogel system experiences small temperature differences, of less 253 than $2^{\circ} \mathrm{C}$, for all times. The temperature increase of the hydrogel is smaller than $10{ }^{\circ} \mathrm{C}$ for all the 254 simulation times. Since the physical properties related to sorption kinetics weakly depend on 
255 temperature, we expect that the small temperature rise and temperature gradients in the system

256 have relatively insignificant impacts on the current system.

\section{References:}

259 (1) Mills, A. F. Heat and Mass Transfer; McGraw-Hill, 1994.

260 (2) Fisher, L. R.; Gamble, R. A.; Middlehurst, J. The Kelvin Equation and the Capillary 261 Condensation of Water. Nature 1981, 290.

262 (3) Yoon, J.; Cai, S.; Suo, Z.; Hayward, R. C. Poroelastic Swelling Kinetics of Thin Hydrogel Layers: Comparison of Theory and Experiment. Soft Matter 2010, 6 (23), 6004-6012.

264 (4) Reuge, N.; Fede, P.; Berthoumieu, J.-F.; Foucoin, F.; Simonin, O. Modeling of the Conditions and of the Turbulence Intensity. J. Appl. Meteorol. Climatol. 2017, 56 (2), $249-261$.

(5) Ponomarenko, I. V.; Glaznev, I. S.; Gubar, A. V.; Aristov, Y. I.; Kirik, S. D. Synthesis and Water Sorption Properties of a New Composite "CaCl2 Confined into SBA-15 Pores." Microporous Mesoporous Mater. 2010, 1-2 (129), 243-250.

273 (7) Wang, Q.; Zhao, L.; Li, C.; Cao, Z. The Decisive Role of Free Water in Determining 274 Homogenous Ice Nucleation Behavior of Aqueous Solutions. Sci. Reports 201661 2016, 
$6(1), 1-8$.

276

277

278

279

280

281

282

283

284

285

286

287

288

289

290

291

292

293

294
(8) Xu, J.; Li, T.; Yan, T.; Wu, S.; Wu, M.; Chao, J.; Huo, X.; Wang, P.; Wang, R. Ultrahigh Solar-Driven Atmospheric Water Production Enabled by Scalable Rapid-Cycling Water Harvester with Vertically Aligned Nanocomposite Sorbent. Energy Environ. Sci. 2021, 14 (11), 5979-5994.

(9) Li, R.; Shi, Y.; Alsaedi, M.; Wu, M.; Shi, L.; Wang, P. Hybrid Hydrogel with High Water Vapor Harvesting Capacity for Deployable Solar-Driven Atmospheric Water Generator. Environ. Sci. Technol. 2018, 52 (19), 11367-11377.

(10) MARSHALL, T. J. THE DIFFUSION OF GASES THROUGH POROUS MEDIA. J. Soil Sci. 1959, 10 (1), 79-82.

(11) Tartakovsky, D. M.; Dentz, M. Diffusion in Porous Media: Phenomena and Mechanisms. Transp. Porous Media 2019, 130 (1), 105-127.

(12) Bouklas, N.; Huang, R. Swelling Kinetics of Polymer Gels: Comparison of Linear and Nonlinear Theories. Soft Matter 2012, 8 (31), 8194-8203.

(13) Subramani, R.; Izquierdo-Alvarez, A.; Bhattacharya, P.; Meerts, M.; Moldenaers, P.; Ramon, H.; Van Oosterwyck, H. The Influence of Swelling on Elastic Properties of Polyacrylamide Hydrogels. Front. Mater. 2020, 7, 212.

(14) Louf, J. F.; Datta, S. S. Poroelastic Shape Relaxation of Hydrogel Particles. Soft Matter 2021, 17 (14), 3840-3847.

(15) Poly(acrylamide) https://polymerdatabase.com/polymers/polyacrylamide.html (accessed 
296 (16) Cummins, B. M.; Chinthapatla, R.; Ligler, F. S.; Walker, G. M. Time-Dependent Model 297 for Fluid Flow in Porous Materials with Multiple Pore Sizes. Anal. Chem. 2017, 89 (8), $4377-4381$.

299 (17) Li, R.; Shi, Y.; Wu, M.; Hong, S.; Wang, P. Photovoltaic Panel Cooling by Atmospheric Water Sorption-Evaporation Cycle. Nat. Sustain. 2020, 3 (8), 636-643.

(18) Conde, M. R. Properties of Aqueous Solutions of Lithium and Calcium Chlorides: Formulations for Use in Air Conditioning Equipment Design. Int. J. Therm. Sci. 2004, 43 (4), 367-382.

304 (19) Bergman, T.; Adrienne, L.; Incropera, F.; DeWitt, D. Fundamentals of Heat and Mass Transfer, 8th Edition | Wiley; John Wiley and Sons: Nashville, TN, 2017. 